\title{
Comparison of Fine-Needle Aspiration and Core Needle Biopsy under Ultrasonographic Guidance for Detecting Malignancy and for the Tissue-Specific Diagnosis of Salivary Gland Tumors
}

\author{
H.-J. Eom, J.H. Lee, M.-S. Ko, Y.J. Choi, R.G. Yoon, K.J. Cho, S.Y. Nam, and J.H. Baek
}

\begin{abstract}
BACKGROUND AND PURPOSE: Diagnostic test accuracy studies for ultrasonography-guided fine-needle aspiration and ultrasonography-guided core needle biopsy have shown inconclusive results due to their heterogenous study designs. Our aim was to compare the diagnostic accuracy of ultrasonography-guided fine-needle aspiration versus ultrasonography-guided core needle biopsy for detecting malignant tumors of the salivary gland and for the tissue-specific diagnosis of salivary gland tumors in a single tertiary hospital.
\end{abstract}

MATERIALS AND METHODS: This retrospective study was approved by our institutional review board and informed consent was waived. Four hundred twelve patients who underwent ultrasonography-guided fine-needle aspiration $(n=155)$ or ultrasonography-guided core needle biopsy ( $n=257$ ) with subsequent surgical confirmation or clinical follow-up were enrolled. We compared the diagnostic accuracy of ultrasonography-guided fine-needle aspiration and ultrasonography-guided core needle biopsy regarding malignant salivary gland tumors and the correct tissue-specific diagnosis of benign and malignant tumors. We also tested the difference between these procedures according to the operator's experience and lesion characteristics.

RESULTS: The inconclusive rates of ultrasonography-guided fine-needle aspiration and ultrasonography-guided core needle biopsy were $19 \%$ and $4 \%$, respectively $(P<.001)$. The overall accuracy of ultrasonography-guided core needle biopsy for diagnosing malignant tumors was significantly higher than that of ultrasonography-guided fine-needle aspiration $(P=.024)$. The correct tissue-specific diagnosis rates of ultrasonography-guided fine-needle aspiration and ultrasonography-guided core needle biopsy were $95 \%$ versus $97 \%$ for benign tumors ( $P=.648)$ and $67 \%$ versus $80 \%$ for malignant tumors $(P=.310)$. Trainees showed significantly lower accuracy with ultrasonography-guided fine-needle aspiration than with ultrasonography-guided core needle biopsy for diagnosing malignant tumors $(P=.021)$. There was no difference between the diagnostic accuracy of ultrasonography-guided fine-needle aspiration and ultrasonography-guided core needle biopsy according to the internal composition of the lesions. There were no complications requiring intervention or hospitalization in our patients.

CONCLUSIONS: Ultrasonography-guided core needle biopsy is superior to ultrasonography-guided fine-needle aspiration in detecting and characterizing malignant tumors of the salivary gland and could emerge as the diagnostic method of choice for patients presenting with a salivary gland mass.

ABBREVIATIONS: CNB = core needle biopsy; FNA = fine-needle aspiration; US = ultrasonography; USCNB = ultrasonography-guided core needle biopsy; USFNA = ultrasonography-guided fine-needle aspiration

T he preoperative diagnosis of salivary gland masses is important for avoiding unnecessary surgery for nonmalignant lesions, and for therapeutic planning for malignant tumors. Fineneedle aspiration (FNA) is a well-accepted and widely used technique for the preoperative diagnosis of salivary gland masses, showing an average specificity for detecting malignant tumors

Received September 28, 2014; accepted after revision November 25. From the Department of Radiology and Research Institute of Radiology (H.-J.E., J.H.L., Y.J.C., R.G.Y., J.H.B.) and Departments of Health Medicine (M.-S.K.), Pathology (K.J.C.), and Otolaryngology (S.Y.N.), Asan Medical Center, University of Ulsan College of Medicine, Seoul, Korea.

Paper previously presented at: Annual Meeting of the Radiological Society of North America, November 30-December 5, 2014; Chicago, Illinois. among these masses of up to $96 \%$ with little variation from study to study. ${ }^{1,2}$ Remarkable variation is seen in the sensitivity of FNA, however, which ranges from $33 \%$ to $100 \%$, with an average of $79 \%{ }^{1-7}$ A recently published meta-analysis has suggested the influence of verification bias on the reported diagnostic accuracies of FNA, leading to an underestimation of its sensitivity and overestimation of its specificity in previous articles. ${ }^{8}$ In addition to verification bias, differences in test conditions have a major effect

Please address correspondence to Jeong Hyun Lee, MD, PhD, Department of Radiology and Research Institute of Radiology, University of Ulsan College of Medicine, Asan Medical Center, 86 Asanbyeongwon-Gil, Songpa-Gu, Seoul 138-736, Republic of Korea; e-mail: jeonghlee@amc.seoul.kr

http://dx.doi.org/10.3174/ajnr.A4247 
on the diagnostic performance of FNA. These test conditions include the use of ultrasonography (US) guidance, the presence of a pathologist on site, the experience level of the operator and the pathologist, and the internal composition of the biopsied masses. For example, US guidance can increase the diagnostic performance of FNA compared with palpation guidance, as evidenced in the diagnosis of thyroid nodules. ${ }^{9}$

Core needle biopsy (CNB) under US guidance is used frequently for masses in the head and neck area ${ }^{10-12}$ and has several advantages over either palpation-guided or US-guided FNA, including the ability to obtain a larger specimen with preserved tissue architecture for possible immunohistochemical staining. This potentially contributes to the low nondiagnostic rate and high average sensitivity (92\%) and specificity (100\%) of this procedure, with little variation in its ability to detect malignant tumors in salivary glands. ${ }^{13}$ Despite the advantages of CNB, its routine use for salivary gland tumors has been debated due to potential morbidity, such as facial nerve damage, use of local anesthesia, and patient discomfort.

A recent meta-analysis was performed to compare the diagnostic performance of FNA and CNB. The authors of that study could not reach a definitive conclusion, however, due to the significant heterogeneity regarding FNA findings in the literature, even with a systematic review. ${ }^{1,14}$ Therefore, a study with a large series that considers different test conditions and minimizes any verification bias is still required for comparing the diagnostic performance of FNA and CNB and ultimately suggesting some clinical guidelines. Accordingly, we designed our current retrospective study to evaluate the diagnostic performance of FNA and $\mathrm{CNB}$, both performed under US guidance, for detecting malignancy and undertaking tissue-specific diagnoses of salivary gland tumors in a large series of patients in our tertiary hospital. We also evaluated the factors influencing the diagnostic performance of both techniques, including the experience level of the operator and the internal composition of the biopsied mass.

\section{MATERIALS AND METHODS Patient Population}

From 2003 to 2012, 474 consecutive patients underwent ultrasonography-guided fine-needle aspiration (USFNA) $(n=192)$ or ultrasonography-guided core needle biopsy (USCNB) $(n=282)$ for a salivary gland mass at our hospital. We retrospectively reviewed the medical records, US images, and the radiologic, cytologic, and/or histologic reports of USFNA and USCNB in all of these patients. After the exclusion of 62 patients (37 from the USFNA group and 25 from the USCNB group) due to limited medical records beyond 1-year clinical follow-up, we finally enrolled 155 patients who underwent USFNA and 257 who underwent USCNB in our study cohorts. There were 217 males and 195 females with an age range of 9-86 years in this series (mean, 53 years).

\section{USFNA and USCNB}

US examinations were performed by using 1 of 3 systems: an iU22 or HDI-5000 U (Philips Healthcare, Best, the Netherlands) or an EUB-7500 (Hitachi Medical Systems, Tokyo, Japan). Each system was equipped with a linear high-frequency probe $(5-14 \mathrm{MHz})$.
After the US examination, the operator performed USFNA or USCNB in accordance with the operator's discretion or clinician's preferences. During image analysis, masses were classified as solid or predominantly solid (proportion of solid component $>50 \%$ ) and cystic or predominantly cystic (proportion of cystic change $>50 \%$ ) according to their internal architecture. All of the examinations, including US-guided biopsy, were performed by one of the faculty members (J.H.L. with US-guided biopsy experience of 14 years or J.H.B. with 18 years' experience) or by a trainee with $<5$ years of US-guided biopsy experience.

Before the procedures, written consent was obtained from the patient. USFNA was performed by using a 23-ga needle attached to a $10-\mathrm{mL}$ disposable plastic syringe with a combination of capillary action and suction-aspiration techniques, depending on the lesion characteristics. Specimens were immediately fixed in 95\% ethanol. USCNB was performed by using disposable 18-ga double-action spring-activated needles (1.1- or 1.6-cm excursion; AceCut; TSK, Tochigi, Japan) or 18-ga dual-action semiautomatic needles (1.0- or 2.0-cm excursion; Stericut with a coaxial guide; TSK Laboratory) after local anesthesia with $2 \%$ lidocaine. Local anesthetic was injected slowly into the subcutaneous fat and just beneath the capsule. Operators selected biopsy needles with adequate throw lengths compared with the maximum length of the target lesions. They were cautioned to put the specimen notch of the inner stylet within the target to minimize the risk of facial nerve damage. When the target was smaller than the length of the specimen notch, the needle was inserted in a caudocranial direction and the superior end of the specimen notch did not pass through the upper top of the target. In cases of cystic or predominantly cystic masses, lesions were biopsied after aspiration of cystic content, and targeting was directed at the solid component inside the mass. Specimens were immediately fixed in a $10 \%$ formalin solution. A freehand technique was used throughout the procedure to achieve accurate mass targeting. A coaxial technique was not used in all cases. All biopsy specimens were evaluated with the naked eye immediately after the procedure for the presence of whitish tissue material to assess the adequacy of the material. If the specimen was insufficient, the USFNA or USCNB was repeated. After the biopsy, firm local compression of the biopsy site with an ice pack was applied for 10-20 minutes.

\section{Histologic Diagnosis and Statistical Analysis}

Biopsy specimens were sent to the pathology department and reviewed by an experienced cytopathologist. A final diagnosis was established on the basis of surgical histologic findings for the surgical group or the USFNA result combined with clinical and/or radiologic follow-up results of $>1$ year in patients who did not undergo surgery. We strictly defined the inconclusive results of USFNA and USCNB. In addition to the commonly used definition of inadequate FNA results ( 6 groups each with $\geq 10$ epithelial cells), ${ }^{15}$ we included indeterminate cytologic results of both USFNA and USCNB among the inconclusive findings when they were insufficient to determine whether the lesion was neoplastic or malignant.

Statistical analysis was performed to compare any difference in the inconclusive rates between USFNA and USCNB. We calculated the sensitivities, specificities, positive and negative predic- 
Table 1: Demographic data and results of US image analysis ${ }^{a}$

\begin{tabular}{lcccc}
\hline & $\begin{array}{c}\text { Total } \\
(\boldsymbol{N}=\mathbf{4 1 2})\end{array}$ & $\begin{array}{c}\text { FNA } \\
(\boldsymbol{n}=155)\end{array}$ & $\begin{array}{c}\text { CNB } \\
(\boldsymbol{n}=\mathbf{2 5 7 )})\end{array}$ & $\boldsymbol{P}$ Value \\
\hline Age $(\mathrm{yr})^{\mathrm{b}}$ & $53 \pm 16$ & $55 \pm 15$ & $52 \pm 16$ & .084 \\
Size $(\mathrm{cm})^{\mathrm{b}}$ & $2.2 \pm 1.1$ & $2.2 \pm 1.2$ & $2.2 \pm 1.1$ & .694 \\
Sex & & & & .374 \\
$\quad$ Male & 217 & $86(55)$ & $131(51)$ & \\
$\quad$ Female & 195 & $69(45)$ & $126(49)$ & \\
Location & & & & .637 \\
$\quad$ Parotid gland & 311 & $119(77)$ & $192(75)$ & \\
$\quad$ Submandibular gland & 101 & $36(23)$ & $65(25)$ & \\
No. of needle passes & & & & .052 \\
$\quad 1$ & 267 & $112(72)$ & $155(60)$ & \\
2 & 132 & $37(24)$ & $95(37)$ & \\
3 & 13 & $6(4)$ & $7(3)$ & \\
\hline
\end{tabular}

a Unless otherwise indicated, data show the number of lesions, with percentages in parentheses.

b Data show means.

Table 2: Summary of inconclusive results of USFNA and USCNB

\begin{tabular}{lcccc}
\hline & Total & Benign & Malignant & FU loss \\
\hline USFNA & 30 & & & \\
$\quad$ Inadequate specimen & 14 & 8 & 1 & 5 \\
$\quad$ Others $^{\mathrm{a}}$ & 16 & 11 & 5 & 0 \\
USCNB $^{\text {Inadequate specimen }}$ & 10 & & & \\
$\quad$ Others $^{\mathrm{a}}$ & 9 & 0 & 1 & 0 \\
\hline
\end{tabular}

Note:-FU indicates follow-up.

a "Others" include inconclusive results for differential diagnoses between benign and malignant salivary gland tumors.

tive values, and overall diagnostic accuracies of USFNA and USCNB for detecting malignant tumors of the salivary gland and for correct tissue-specific diagnoses. We also tested any difference in the diagnostic profile of USFNA and USCNB. For comparison of tissue-specific diagnosis rates between 2 modalities, we excluded false-positive and false-negative results of USFNA and USCNB and those confirmed as benign by clinical and/or radiologic follow-up from the statistical analysis. Statistical testing was also performed to identify any differences between USFNA and USCNB according to the experience level of the operator and the internal composition of the biopsied lesions.

Statistical analysis was performed by using the SPSS software package (Version 19.0 for Windows; IBM, Armonk, New York). Continuous variables were compared by using an unpaired Student $t$ test or Mann-Whitney $U$ test, depending on the data distribution. Categoric variables were expressed by using frequencies and percentages and were tested by using a $\chi^{2}$ or Fisher exact test. A $P$ value of $<.05$ was statistically significant.

\section{RESULTS}

Patient demographic data and the results of the US image analysis are summarized in Table 1. There were no significant differences between the USFNA and USCNB groups with respect to demographic data, the location and size of the lesions, internal composition on US, and the number of needle passes. The inconclusive rates were 19\% (30/155) for USFNA and 4\% (10/257) for USCNB, which was a significant difference $(P<.001)$. The summary of inconclusive results of USFNA and USCNB is shown in Table 2. The final diagnoses of USFNA and USCNB are detailed in Fig 1. Among the tumors that were classified as benign $(n=76)$ and malignant $(n=19)$ by USFNA, there were 5 false-negative and 3 false-positive results for the diagnosis of a malignant tumor of the salivary gland. Among the tumors that were classified as benign ( $n=177)$ and malignant $(n=53)$ by USCNB, there were 4 falsenegative results and 1 false-positive result. The false-positive and false-negative results are detailed in Table 3. There were no major complications among our study patients needing medication or hospitalization according to a review of the electronic medical records and radiologic reports.

The diagnostic accuracies of USFNA and USCNB in our current evaluation are summarized in Table 4 . The sensitivity, specificity, accuracy, and positive and negative predictive values of USCNB for detecting malignant salivary gland tumors tended to be higher than those of USFNA. However, our statistical analysis revealed significance only for diagnostic accuracy $(P=.024)$. The tissue-specific diagnosis rates of USFNA were 95\% (39/41) in benign tumors and $67 \%(10 / 15)$ in malignant tumors; those of USCNB were $97 \%(142 / 147)$ in benign tumors and 80\% (32/40) in malignant tumors. There were no significant differences in the tissue-specific diagnosis rates of either benign $(P=.648)$ or malignant $(P=.310)$ tumors between the USFNA and USCNB groups.

Regarding the experience level of the operators, the sensitivity, specificity, accuracy, and positive and negative predictive values of USCNB for diagnosing malignant tumors tended to be higher than those of USFNA for both faculty members and trainees. However, when the diagnostic procedures were performed by trainees, the diagnostic accuracy of USFNA was significantly lower than that of USCNB $(P=.021)$. The sensitivity, specificity, accuracy, and positive and negative predictive values of USCNB for diagnosing malignant tumors were not significantly higher than those of USFNA, regardless of the internal composition. These results are summarized in Tables 5 and 6 .

\section{DISCUSSION}

The results of our current study can be summarized as follows: First, USCNB shows significantly better diagnostic performance-expressed as diagnostic accuracy and inconclusive rates - than USFNA for detecting malignant salivary gland tumors; second, the diagnostic accuracy of USFNA is significantly affected by the operator's experience level, whereas that of USCNB is not; third, USFNA and USCNB do not show significantly different tissue-specific diagnosis rates; and finally, the diagnostic performances of these methods are not affected by the internal architecture of a biopsied lesion.

Our current study findings confirm the superiority of USCNB over USFNA in terms of diagnostic accuracy and inconclusive rates for detecting malignant salivary gland tumors. The inconclusive rates of USFNA and USCNB in our study were 19\% and $4 \%$, respectively. The cytologic results were considered inconclusive when the specimen was deemed inadequate for making a diagnosis or as indeterminate for the presence of malignant tumor or neoplasm when the USFNA or USCNB results were insufficient to make a clinical decision and management plan, both of which are crucial for proper patient management. It is quite disappointing that nearly one-fifth of USFNA examination findings were inconclusive from a practical point of view.

The variability in the diagnostic accuracy of previously re- 


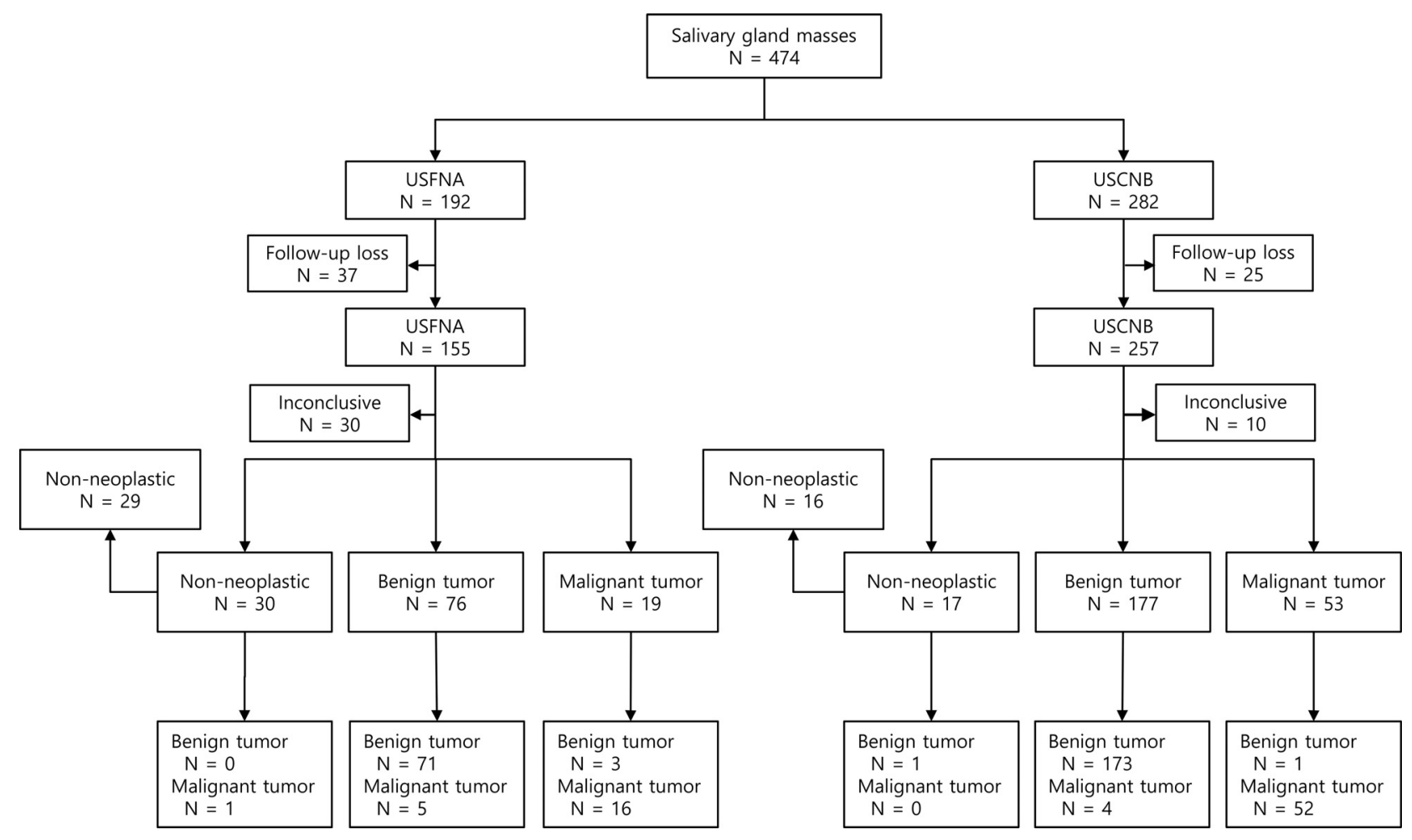

FIG 1. The results of USFNA and USCNB in all patients.

Table 3: False-positive and false-negative results of USFNA and USCNB in salivary gland tumors

\begin{tabular}{ll}
\hline \multicolumn{1}{c}{ Biopsy Results } & \multicolumn{1}{c}{ Final Pathologies } \\
\hline USFNA & \\
False-negative & Mucoepidermoid carcinoma \\
Warthin tumor & Mucoepidermoid carcinoma \\
Pleomorphic adenoma & Mucoepidermoid carcinoma \\
Pleomorphic adenoma & Carcinoma ex pleomorphic adenoma \\
Pleomorphic adenoma & Basal cell adenocarcinoma \\
Basal cell adenoma & \\
False-positive & Warthin tumor with squamous metaplasia \\
Squamous cell carcinoma & Oncocytoma \\
Acinic cell carcinoma & Oncocytoma \\
Acinic cell carcinoma & \\
USCNB & \\
False-negative & Epithelial-myoepithelial carcinoma \\
Pleomorphic adenoma & Carcinoma ex pleomorphic adenoma \\
Pleomorphic adenoma & Carcinoma ex pleomorphic adenoma \\
Pleomorphic adenoma & Basal cell adenocarcinoma \\
Basal cell adenoma & \\
False-positive & Pleomorphic adenoma \\
Low-grade mucoepidermoid carcinoma &
\end{tabular}

icant heterogeneity. ${ }^{14}$ The results of USCNB in our study are comparable with those of this latter report.

Our study results also demonstrated that even with US guidance, the diagnostic accuracy of FNA is significantly lower than that of USCNB. This lower diagnostic accuracy could have resulted from the histologic diversity of the salivary gland tumors and could suggest that cytologic examination alone is not sufficient for diagnosing malignant tumors of the salivary glands, thus necessitating a larger specimen with preserved histologic architecture. Schmidt et al ${ }^{1,14}$ tried to compare the diagnostic performances of FNA and CNB for detecting malignant salivary gland tumors in 2 separate meta-analyses. However, the authors stated that a direct comparison of FNA and CNB was not possible be-

ported FNA results is a well-known problem. ${ }^{16}$ This variability was evident in recent studies that evaluated the sensitivity of FNA for distinguishing benign from malignant salivary gland tumors, with sensitivities ranging from $38 \%$ to $87.8 \%$. $^{2,16-18}$ These reports did not state whether US was used for guidance during the biopsy. Our current study suggests that US guidance could yield better diagnostic accuracy than palpation-guided biopsy, given the relatively high diagnostic sensitivity that we observed. On the other hand, a recently published meta-analysis study found that USCNB has an overall sensitivity of 0.92 (95\% CI, 0.77-0.98) and an overall specificity of 1.00 (95\% CI, $0.76-1.00)$, with no signif- cause of an unexpected significant heterogeneity in the findings of studies regarding FNA, and they concluded that CNB could be reserved for special clinical settings, such as cases that were not diagnosed with FNA, given the theoretic drawbacks of CNB, such as facial nerve damage or use of local anesthesia. ${ }^{1,14}$

Our present data demonstrate that USCNB can be safely performed because none of our 282 patients experienced facial nerve injury, and it has a significantly higher diagnostic accuracy for malignant salivary gland tumors. Another disadvantage of USCNB is that it uses larger bore needles compared with USFNA, which might increase the potential risk for tumor seeding along 
Table 4: Comparison of diagnostic accuracies of USFNA and USCNB in salivary gland tumors ${ }^{a}$

\begin{tabular}{llll}
\hline \multicolumn{1}{c}{ Diagnostic Accuracy } & \multicolumn{1}{c}{ USFNA } & \multicolumn{1}{c}{ USCNB } & $\boldsymbol{P}$ Value \\
\hline Sensitivity & $76(16 / 21)$ & $93(52 / 56)$ & .057 \\
Specificity & $96(71 / 74)$ & $99(173 / 174)$ & .081 \\
Accuracy & $92(87 / 95)$ & $98(225 / 230)$ & $.024^{\mathrm{b}}$ \\
Positive predictive value & $84(16 / 19)$ & $98(52 / 53)$ & .054 \\
Negative predictive value & $93(71 / 76)$ & $98(173 / 177)$ & .133 \\
\hline
\end{tabular}

${ }^{a}$ Data show percentages, with patient numbers in parentheses.

b Significant.

\begin{tabular}{|c|c|c|c|}
\hline $\begin{array}{c}\text { Diagnostic } \\
\text { Values }\end{array}$ & FNA (\%) & CNB (\%) & $P$ Value \\
\hline \multicolumn{4}{|l|}{ Sensitivity } \\
\hline Faculty & 79 (15/19) & $89(32 / 36)$ & .426 \\
\hline Trainee & $50(1 / 2)$ & $100(20 / 20)$ & .091 \\
\hline \multicolumn{4}{|l|}{ Specificity } \\
\hline Faculty & $97(57 / 59)$ & $99(96 / 97)$ & .557 \\
\hline Trainee & $93(14 / 15)$ & $100(77 / 77)$ & .163 \\
\hline \multicolumn{4}{|l|}{ Accuracy } \\
\hline Faculty & $92(72 / 78)$ & $96(128 / 133)$ & .336 \\
\hline Trainee & 88 (15/17) & $100(97 / 97)$ & $.021^{b}$ \\
\hline
\end{tabular}

a Data show percentages, with numbers in parentheses.

bignificant.

Table 6: Comparison of diagnostic accuracies of USFNA and USCNB according to the internal component of salivary gland tumors $^{\mathrm{a}}$

\begin{tabular}{clll}
\hline \multicolumn{1}{c}{ Diagnostic Values } & FNA (\%) & CNB (\%) & $P$ Value \\
\hline Sensitivity & & & \\
$\quad$ Solid/predominantly solid & $82(14 / 17)$ & $94(46 / 49)$ & .172 \\
$\quad \begin{array}{c}\text { Cystic/predominantly cystic } \\
\text { Specificity }\end{array}$ & $50(2 / 4)$ & $86(6 / 7)$ & .491 \\
$\quad \begin{array}{l}\text { Solid/predominantly solid } \\
\quad \text { Cystic/predominantly cystic }\end{array}$ & $97(66 / 68)$ & $99(154 / 155)$ & .221 \\
Accuracy & & $100(19 / 19)$ & .240 \\
$\quad$ Solid/predominantly solid & $94(80 / 85)$ & $98(200 / 204)$ & .130 \\
$\quad$ Cystic/predominantly cystic & $70(7 / 10)$ & $96(25 / 26)$ & .057 \\
\hline
\end{tabular}

${ }^{a}$ Data show percentages, with numbers in parentheses.

the needle tract. ${ }^{16}$ In this regard, besides a potential risk of malignant tumor seeding, benign tumors such as pleomorphic adenomas can have problematic recurrences, which may not even be evident until many years after the initial surgery. Although Novoa et $\mathrm{al}^{19}$ recently reviewed the safety of USCNB in the work-up of 438 head and neck lesions with 7 years of clinical follow-up and found no evidence of tumor cell seeding, further clinical investigation of tumor recurrence following USCNB is necessary in a large population during a long time course before the safety of USCNB can be verified and widely accepted.

Another interesting result of our current study is that the diagnostic accuracy of USFNA performed by trainees was significantly lower than that of USCNB performed by trainees. This result suggests that USFNA might be more dependent on the experience level of the operator than USCNB. It also suggests that USFNA is technically more demanding than USCNB and needs accurate localization of a lesion by using both the US probe and the needle, repeated continuous to-and-fro motions of the needle under real-time US monitoring, and an adequate combination of capillary action and suction-aspiration techniques according to the lesion characteristics. ${ }^{1,2,20,21}$
The biopsy of salivary gland lesions carries a risk of complications such as hematoma, infection, or facial nerve injury. The retromandibular vein and external carotid artery are the major intraparotid vessels that are well-visualized with US imaging and can be easily avoided. ${ }^{22}$ However, the facial nerve is not readily identified with US. ${ }^{22,23}$ It has been suggested in previous reports that USFNA carries a lower risk of complications than USCNB. ${ }^{13,24,25}$ In our current investigation, which included 282 patients who underwent USCNB, there were no major complications observed such as facial nerve injury. Our suggestions for reducing the risk of potential facial nerve damage with USCNB are to select a core needle device with an adequate throw length compared with the maximum length of the targets and to obtain the specimen while carefully keeping the specimen notch within the target without passing through the upper margin of the target. Our study results confirm that facial nerve damage can generally be avoided by performing USCNB with caution.

In addition to the inherent limitations related to a retrospective study, our study had several other limitations. The use of USFNA and USCNB was not randomized (randomization would have been ideal), and the operator performed USFNA or USCNB in accordance with his or her discretion or the clinician's preferences. However, because our study showed no significant differences in evaluated clinical and US imaging data between USFNA and USCNB lesions, we think that bias by operators/clinicians as to whether USFNA or USCNB was used is limited. Additionally, although we demonstrated that USCNB is a safe procedure without major complications for the histologic diagnosis of salivary gland tumors, we did not compare the degree of patient discomfort from the use of lidocaine anesthesia or the larger bore needle used for USCNB with that used for USFNA. Patient discomfort might thus be the subject of future prospective studies.

\section{CONCLUSIONS}

USCNB is superior to USFNA in that it has a lower inconclusive rate and higher diagnostic accuracy for detecting malignant salivary gland tumors. USCNB could be considered the US-guided biopsy procedure of choice for evaluating patients with a salivary gland mass because it can aid in appropriate therapeutic planning and minimize unnecessary repeat biopsies.

\section{REFERENCES}

1. Schmidt RL, Hall BJ, Wilson AR, et al. A systematic review and metaanalysis of the diagnostic accuracy of fine-needle aspiration cytology for parotid gland lesions. Am J Clin Pathol 2011;136:45-59

2. Balakrishnan K, Castling B, McMahon J, et al. Fine needle aspiration cytology in the management of a parotid mass: a two centre retrospective study. Surgeon 2005;3:67-72

3. Aversa S, Ondolo C, Bollito E, et al. Preoperative cytology in the management of parotid neoplasms. Am J Otolaryngol 2006;27: 96-100

4. Seethala RR, LiVolsi VA, Baloch ZW. Relative accuracy of fine-needle aspiration and frozen section in the diagnosis of lesions of the parotid gland. Head Neck 2005;27:217-23

5. Al Salamah SM, Khalid K, Khan IA, et al. Outcome of surgery for parotid tumours: 5-year experience of a general surgical unit in a teaching hospital. ANZ J Surg 2005;75:948-52

6. Al-Khafaji BM, Nestok BR, Katz RL. Fine-needle aspiration of $\mathbf{1 5 4}$ parotid masses with histologic correlation: ten-year experience at 
the University of Texas M.D. Anderson Cancer Center. Cancer 1998;84:153-59

7. Schelkun PM, Grundy WG. Fine-needle aspiration biopsy of head and neck lesions. J Oral Maxillofac Surg 1991;49:262-67

8. Schmidt RL, Jedrzkiewicz JD, Allred RJ, et al. Verification bias in diagnostic accuracy studies for fine- and core needle biopsy of salivary gland lesions in otolaryngology journals: a systematic review and analysis. Head Neck 2014;36:1654-61

9. Izquierdo R, Arekat MR, Knudson PE, et al. Comparison of palpation-guided versus ultrasound-guided fine-needle aspiration biopsies of thyroid nodules in an outpatient endocrinology practice. Endocr Pract 2006;12:609-14

10. Hakala T, Kholova I, Sand J, et al. A core needle biopsy provides more malignancy-specific results than fine-needle aspiration biopsy in thyroid nodules suspicious for malignancy. J Clin Pathol 2013;66:1046-50

11. Samir AE, Vij A, Seale MK, et al. Ultrasound-guided percutaneous thyroid nodule core biopsy: clinical utility in patients with prior nondiagnostic fine-needle aspirate. Thyroid 2012;22:461-67

12. Burke $C$, Thomas $R$, Inglis $C$, et al. Ultrasound-guided core biopsy in the diagnosis of lymphoma of the head and neck: a 9-year experience. Br J Radiol 2011;84:727-32

13. Taki S, Yamamoto T, Kawai A, et al. Sonographically guided core biopsy of the salivary gland masses: safety and efficacy. Clin Imaging 2005;29:189-94

14. Schmidt RL, Hall BJ, Layfield LJ. A systematic review and meta-analysis of the diagnostic accuracy of ultrasound-guided core needle biopsy for salivary gland lesions. Am J Clin Pathol 2011;136:516-26

15. Goellner JR, Gharib H, Grant CS, et al. Fine needle aspiration cytology of the thyroid, 1980 to 1986. Acta Cytol 1987;31:587-90
16. Douville NJ, Bradford CR. Comparison of ultrasound-guided core biopsy versus fine-needle aspiration biopsy in the evaluation of salivary gland lesions. Head Neck 2013;35:1657-61

17. Pitts DB, Hilsinger RL Jr, Karandy E, et al. Fine-needle aspiration in the diagnosis of salivary gland disorders in the community hospital setting. Arch Otolaryngol Head Neck Surg 1992;118:479-82

18. Jayaram N, Ashim D, Rajwanshi A, et al. The value of fine-needle aspiration biopsy in the cytodiagnosis of salivary gland lesions. $\mathrm{Di}$ agn Cytopathol 1989;5:349-54

19. Novoa E, Gurtler N, Arnoux A, et al. Role of ultrasound-guided core-needle biopsy in the assessment of head and neck lesions: a meta-analysis and systematic review of the literature. Head Neck 2012;34:1497-503

20. Huang YC, Wu CT, Lin G, et al. Comparison of ultrasonographically guided fine-needle aspiration and core needle biopsy in the diagnosis of parotid masses. J Clin Ultrasound 2012;40:189-94

21. Colella G, Cannavale R, Flamminio F, et al. Fine-needle aspiration cytology of salivary gland lesions: a systematic review. J Oral Maxillofac Surg 2010;68:2146-53

22. Howlett DC, Menezes LJ, Lewis K, et al. Sonographically guided core biopsy of a parotid mass. AJR Am J Roentgenol 2007;188:223-27

23. Verma K, Kapila K. Role of fine needle aspiration cytology in diagnosis of pleomorphic adenomas. Cytopathology 2002;13:121-27

24. Wan YL, Chan SC, Chen YL, et al. Ultrasonography-guided coreneedle biopsy of parotid gland masses. AJNR Am J Neuroradiol 2004;25:1608-12

25. Kesse KW, Manjaly G, Violaris N, et al. Ultrasound-guided biopsy in the evaluation of focal lesions and diffuse swelling of the parotid gland. Br J Oral Maxillofac Surg 2002;40:384-88 